\title{
Two-colour intensity suppression of multiphoton ionization of atomic deuterium
}

To cite this article: P Bowe et al 1998 J. Phys. B: At. Mol. Opt. Phys. 311003

View the article online for updates and enhancements.

\section{Related content}

A measurement of the two-photon ionization of atomic hydrogen $\mathrm{H}(2 \mathrm{~s})$ near the resonance with the $3 p$ state

Philippe Antoine, Nour-Eddin Essarroukh, Jozo Jureta et al.

Three-photon ionization of $\mathrm{Ca}$

R S Dygdala, K Karasek, F Giammanco et al.

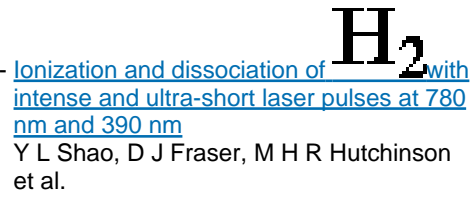

\section{Recent citations}

- Control of a two-colour photoionization
through a time delay between two
components of the laser pulse
J Matulewski et al

\section{IOP ebooks}

Bringing you innovative digital publishing with leading voices to create your essential collection of books in STEM research. Start exploring the collection - download the first chapter of every title for free. 


\title{
Two-colour intensity suppression of multiphoton ionization of atomic deuterium
}

\author{
P Bowe $\dagger \S$, R W O’Neill†, P J M van der Burgt $\dagger$, J A Slevin $\dagger$, \\ A Raczyńskił, J Zarembał and S Chwirot $\ddagger$ \\ $\dagger$ Department of Experimental Physics, National University of Ireland, Maynooth, Co Kildare, \\ Ireland \\ $\ddagger$ Instytut Fizyki, Uniwersytet M Kopernika, ul. Grudziądzka 5-7, PL 87-100 Toruń, Poland
}

Received 17 September 1997, in final form 27 November 1997

\begin{abstract}
We report a strong suppression of the three-photon ionization resonant with the $2 \mathrm{~s}$ state of atomic deuterium at $243 \mathrm{~nm}$ by the introduction of a second colour (486 nm) coupling the $2 \mathrm{~s}$ and $4 \mathrm{p}$ states. This coupling reduces the ionization probability by several orders of magnitude. The experiment is performed using fundamental and second harmonic frequencies of the same laser, with laser intensities up to a maximum value of $88 \mathrm{MW} \mathrm{cm}^{-2}$ and an atomic target density of the order of $5 \times 10^{11} \mathrm{~cm}^{-3}$. The production of both $\mathrm{D}^{+}$ions and $\mathrm{D}(2 \mathrm{~s})$ atoms is measured as a function of the intensity of the fundamental beam $(486 \mathrm{~nm})$. The experimental data are compared with a theoretical calculation using essential atom + field states and taking into account the experimental laser bandwidths.
\end{abstract}

\section{Introduction}

It is well known that excited bound states in atoms are important in multiphoton ionization processes when resonantly excited or coupled during the ionization process. Enhancement or suppression of ion yields can result from population transfers occurring when different atomic levels are shifted into resonance with the laser field. Working in a regime of high laser intensity $\left(\sim 10^{13} \mathrm{~W} \mathrm{~cm}^{-2}\right)$ with ultra-fast laser pulses ( $\sim 100 \mathrm{~s}$ of femtoseconds), Storey et al (1993) and Vrijen et al (1993) controlled multiphoton ionization rates by varying the duration as well as the intensity of the laser pulse. Jones (1995) used two temporally separated, identical laser pulses to observe modulations in the photoionization yield in sodium. De Boer and Muller (1992) used a slow ( $\sim 5 \mathrm{~ns})$ probe laser beam to identify resonant intermediate states in xenon populated by an intense subpicosecond laser. Chen et al (1990) studied the ionization of mercury in a two-colour experiment and observed a modulation of the ionization rate due to interference between the fundamental and third harmonic coupling of the initial and intermediate states. Using a similar two-colour scheme, Protopapas and Knight (1995) have shown theoretically that precise matching of the phase and state coupling parameters can lead to the modulation of the ionization rate with nearly complete contrast. Shao et al (1996) have made an experimental study of coherent effects in several two-colour multiphoton ionization processes in sodium.

Atomic hydrogen has been the focus of several studies of doubly resonant twocolour multiphoton ionization using a $243 \mathrm{~nm}$ pulsed laser to couple the $1 \mathrm{~s}$ and $2 \mathrm{~s}$

$\S$ Present address: Institute of Physics and Astronomy, Aarhus University, DK-8000 Aarhus C, Denmark. 
states through two-photon excitation, in combination with a $656 \mathrm{~nm}$ laser to couple 2s with 3p. From observations of the photoion yield Zhang et al $(1993$, 1995) demonstrated electromagnetically induced transparency in atomic hydrogen at high densities $\left(10^{14}-10^{15} \mathrm{~cm}^{-3}\right)$ and showed how the process can be used for nonlinear optical generation of Lyman- $\beta$ radiation. This process was predicted and modelled by Harris et al (1990) using a perturbative approach where the populations of the ground and metastable states are assumed to be stationary. Sihombing et al (1996) have measured the photoionization signal as a function of the product of atomic density and interaction length. Thompson et al (1995) have measured photoion and Lyman- $\beta$ yields as a function of the intensities of the 243 and $656 \mathrm{~nm}$ lasers, using an atomic hydrogen density of $10^{14} \mathrm{~cm}^{-3}$.

In this paper we report on the strong suppression of the multiphoton ionization rate of atomic deuterium in a doubly resonant two-colour process using fundamental and second harmonic frequencies of the same laser. The second harmonic beam $(243 \mathrm{~nm})$ is used to couple the $1 \mathrm{~s}$ and $2 \mathrm{~s}$ states through two-photon excitation, whereas the fundamental beam $(486 \mathrm{~nm})$ couples $2 \mathrm{~s}$ with $4 \mathrm{p}$. (The harmonic relationship between energy levels which allows the use of a single laser to study this process is unique to hydrogenic systems.) The experiment is performed with relatively low laser powers and long pulse lengths $\left(<10^{8} \mathrm{~W} \mathrm{~cm}^{-2}\right.$ and $\left.\sim 13 \mathrm{~ns}\right)$ and with relatively low atomic densities $\left(\sim 5 \times 10^{11} \mathrm{~cm}^{-3}\right)$. These experimental conditions are such that the process can be accurately treated within a single-particle model in contrast to the experiments of Zhang et al (1993, 1995), Thompson et al (1995) and Sihombing et al (1996), where nonlinear coupling of 3p and 1s was present and where the process was described in terms of the nonlinear susceptibility. In the present experiment the radiative decay of the $4 \mathrm{p}$ state is not observed, but the yield of metastable $2 \mathrm{~s}$ atoms is measured by applying a quenching field in the interaction region after the laser pulse. We develop an essential states model of the interaction of two harmonic laser frequencies with a single deuterium atom in order to model the ionization and we average our theoretical results over the laser bandwidth. We compare our predictions of energy level populations with experimental measurements of the photoion yield and quenched metastable 2s population as a function of the intensity of the fundamental laser.

\section{Theory}

The scheme of relevant energy levels is shown in figure 1. Deuterium is ionized in a threephoton absorption process at $243 \mathrm{~nm}$, resonant with the metastable $2 \mathrm{~s}$ state. The ionization rate, at a particular value of second harmonic $(243 \mathrm{~nm})$ laser intensity, is investigated as a function of the intensity of the fundamental $(486 \mathrm{~nm})$ output from the same laser, which couples the $2 \mathrm{~s}$ level with the $4 \mathrm{p}$ level. From the $4 \mathrm{p}$ level the continuum can be reached by the absorption of a photon of either frequency.

The following theoretical formalism is presented in atomic units. For the sake of convenience (to obtain a time-independent Hamiltonian and to interpret the results directly in terms of multiphoton processes) we use a full quantum description of the two interacting monochromatic laser fields, and describe the evolution of the system in the subspace of the essential atom + field states

$$
|a\rangle=\left|1 \mathrm{~s}, N_{0}, N_{1}\right\rangle \quad|b\rangle=\left|2 \mathrm{~s}, N_{0}-2, N_{1}\right\rangle
$$

and

$$
|c\rangle=\frac{1}{\sqrt{2}}\left(\left|4 \mathrm{p}, m=1, N_{0}-2, N_{1}-1\right\rangle+\left|4 \mathrm{p}, m=-1, N_{0}-2, N_{1}-1\right\rangle\right) .
$$




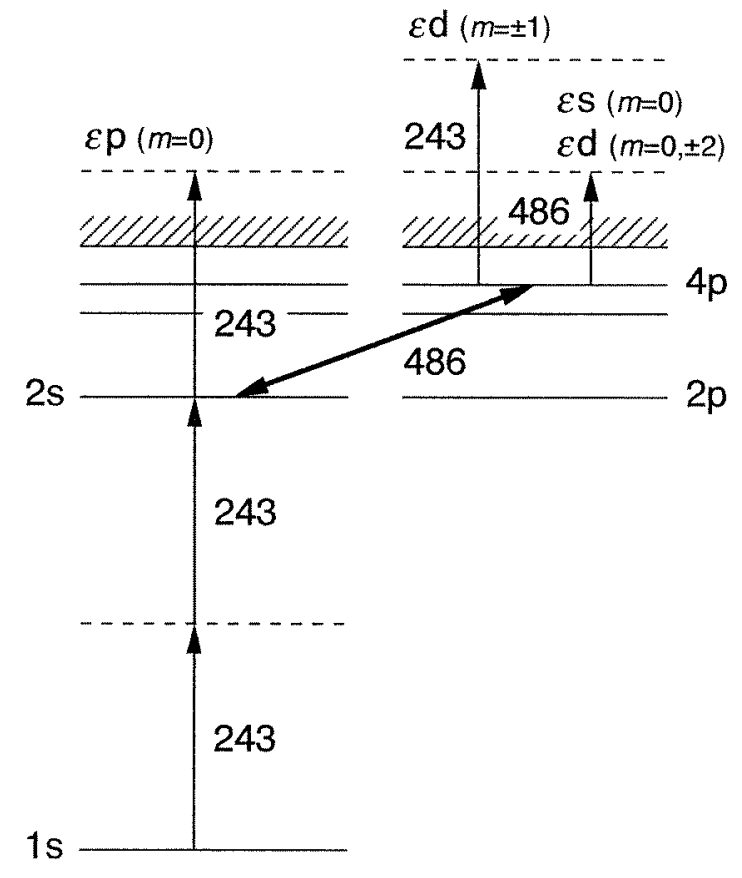

Figure 1. Energy level scheme.

Because the two fields are sufficiently strong $\left(N_{0} \gg 1\right.$ and $\left.N_{1} \gg 1\right)$ to make the laser approximation, we introduce the classical field amplitudes $\varepsilon_{0}$ and $\varepsilon_{1}$, the squares of which are proportional to $N_{0}$ and $N_{1}$, respectively. The subscripts 0 and 1 refer to the second harmonic photons $(243 \mathrm{~nm})$ and the fundamental photons $(486 \mathrm{~nm})$, respectively. The frequencies of both fields are set at the resonant values for two-photon excitation of $1 \mathrm{~s}-2 \mathrm{~s}$ and single-photon excitation of $2 \mathrm{~s}-4 \mathrm{p}$. The fundamental and second harmonic fields have linear polarizations that are mutually orthogonal.

The states $|a\rangle$ and $|b\rangle$ are coupled by a second-order non-resonant coupling $U$ via the virtual state

$$
|j\rangle=\left|2 \mathrm{p}, m=0, N_{0}-1, N_{1}\right\rangle .
$$

The four-photon coupling of the $1 \mathrm{~s}$ and $2 \mathrm{~s}$ states by the fundamental beam is assumed to be negligible due to the small generalized four-photon cross section, and hence interference effects are not significant. The fundamental beam couples the $2 \mathrm{~s}$ state with the $4 \mathrm{p}$ state. From the $4 \mathrm{p}$ state the continuum can be reached by the absorption of a photon of either frequency, as shown in figure 1, and accordingly seven continuum states are used in our calculations. Continuum-continuum coupling is not significant at our laser intensities and is not included.

The matrix elements of the atom-field couplings $U$ and $V$, evaluated in the dipole approximation in the length gauge, are $V_{b c}=0.370 \varepsilon_{1}$ and $U_{a b}=2.98 \varepsilon_{0}^{2}$ au, respectively. The bound-continuum matrix elements, calculated for fixed continuum energy values defined through energy conservation (pole approximation), lead to the following widths of $|b\rangle$ and $|c\rangle$ :

$$
\Gamma_{b}=6.41 \varepsilon_{0}^{2} \quad \Gamma_{c}=7.43 \varepsilon_{1}^{2}+0.388 \varepsilon_{0}^{2} .
$$


The shifts of the levels $|b\rangle$ and $|c\rangle$ are proportional to the squares of the field amplitudes and can be neglected compared to the first-order shift of these levels due to their direct coupling by the field $\varepsilon_{1}$. The induced widths are of the same order of magnitude as the shifts, but they are responsible for a qualitatively new effect of a decay to the continuum.

The probability amplitudes $\alpha(t), \beta(t)$ and $\gamma(t)$ of finding the system in the states $|a\rangle$, $|b\rangle$ and $|c\rangle$, respectively, after time $t$ can be evaluated exactly using the resolvent technique to give

$$
\begin{aligned}
\alpha(t) & =\frac{1}{2 \pi \mathrm{i}} \int_{\infty+\mathrm{i} \eta}^{-\infty+\mathrm{i} \eta} \mathrm{d} z \mathrm{e}^{-\mathrm{i} z t} \frac{\left(z+\frac{1}{2} \mathrm{i} \Gamma_{b}\right)\left(z+\frac{1}{2} \mathrm{i} \Gamma_{c}\right)-V_{b c}^{2}}{\left(z-z_{1}\right)\left(z-z_{2}\right)\left(z-z_{3}\right)} \\
& =\sum_{j=1}^{3} \mathrm{e}^{-\mathrm{i} z_{j} t} \frac{\left(z_{j}+\frac{1}{2} \mathrm{i} \Gamma_{b}\right)\left(z_{j}+\frac{1}{2} \mathrm{i} \Gamma_{c}\right)-V_{b c}^{2}}{\prod_{k \neq j}\left(z_{j}-z_{k}\right)} \\
\beta(t) & =\frac{1}{2 \pi \mathrm{i}} \int_{\infty+\mathrm{i} \eta}^{-\infty+\mathrm{i} \eta} \mathrm{d} z \mathrm{e}^{-\mathrm{i} z t} \frac{U_{b a}\left(z+\frac{1}{2} \mathrm{i} \Gamma_{c}\right)}{\left(z-z_{1}\right)\left(z-z_{2}\right)\left(z-z_{3}\right)}=\sum_{j=1}^{3} \mathrm{e}^{-\mathrm{i} z_{j} t} \frac{U_{b a}\left(z_{j}+\frac{1}{2} \mathrm{i} \Gamma_{c}\right)}{\prod_{k \neq j}\left(z_{j}-z_{k}\right)} \\
\gamma(t) & =\frac{1}{2 \pi \mathrm{i}} \int_{\infty+\mathrm{i} \eta}^{-\infty+\mathrm{i} \eta} \mathrm{d} z \mathrm{e}^{-\mathrm{i} z t} \frac{V_{c b} U_{b a}}{\left(z-z_{1}\right)\left(z-z_{2}\right)\left(z-z_{3}\right)}=\sum_{j=1}^{3} \mathrm{e}^{-\mathrm{i} z_{j} t} \frac{V_{c b} U_{b a}}{\prod_{k \neq j}\left(z_{j}-z_{k}\right)}
\end{aligned}
$$

where $\left(z-z_{1}\right)\left(z-z_{2}\right)\left(z-z_{3}\right)$ is the determinant of the matrix

$$
\left(\begin{array}{ccc}
z & -U_{a b} & 0 \\
-U_{b a} & z+\frac{1}{2} \mathrm{i} \Gamma_{b} & -V_{b c} \\
0 & -V_{c b} & z+\frac{1}{2} \mathrm{i} \Gamma_{c}
\end{array}\right) .
$$

The total ionization probability is $P_{\mathrm{on}}=1-|\alpha(t)|^{2}-|\beta(t)|^{2}-|\gamma(t)|^{2}$.

Figure 2 shows our calculated occupation probabilities for monochromatic, rectangular laser pulses of duration $24.2 \mathrm{~ns}=10^{9}$ au. The amplitude $\varepsilon_{0}$ of the second harmonic field is kept constant at $8 \times 10^{-5}$ au $\left(2.25 \times 10^{8} \mathrm{~W} \mathrm{~cm}^{-2}\right)$, while the amplitude $\varepsilon_{1}$ of the fundamental field is varied from $1 \times 10^{-9}$ to $1 \times 10^{-4}$ au $\left(3.5 \times 10^{2}-3.5 \times 10^{8} \mathrm{~W} \mathrm{~cm}^{-2}\right)$. In the absence of the fundamental field a second harmonic field amplitude of $8 \times 10^{-5}$ au is sufficient to produce an ionization probability essentially equal to unity.

Our calculations show that at high fundamental amplitudes, ionization is completely suppressed and there is essentially complete population trapping in the ground state. The populations of the $2 \mathrm{~s}$ and $4 \mathrm{p}$ states increase at moderate amplitudes and decrease at higher amplitudes. The ionization probability through the $(2+1)$-photon channel, initially almost unity, decreases by seven orders of magnitude for a corresponding change of less than three orders of magnitude in the fundamental amplitude. The ionization from $4 p$ by a fundamental photon increases for medium coupling fields and saturates at a low population probability. This is due to the fact that the reduction in the population of the $4 \mathrm{p}$ state is compensated by the greater probability of ionization out of that state. However, the ionization from $4 p$ by a second harmonic photon provides no such compensation and hence rises and falls with the population of the $4 \mathrm{p}$ level. These marked changes in transition probabilities are due to the Autler-Townes splitting (Autler and Townes 1955) of the initially degenerate states $|b\rangle$ and $|c\rangle$ which results in a loss of the resonant character of the transition. The resonance can be restored and the ionization probability brought back to a high level by detuning the laser and adjusting the frequency to the shifted level. It is also important to stress that the details of the bound states populations of figure 1 depend on the duration of the rectangular pulse used in our calculations. This is connected with the Rabi-type (damped) oscillations of the populations, which may still be important at the instant of switching the field off, at 


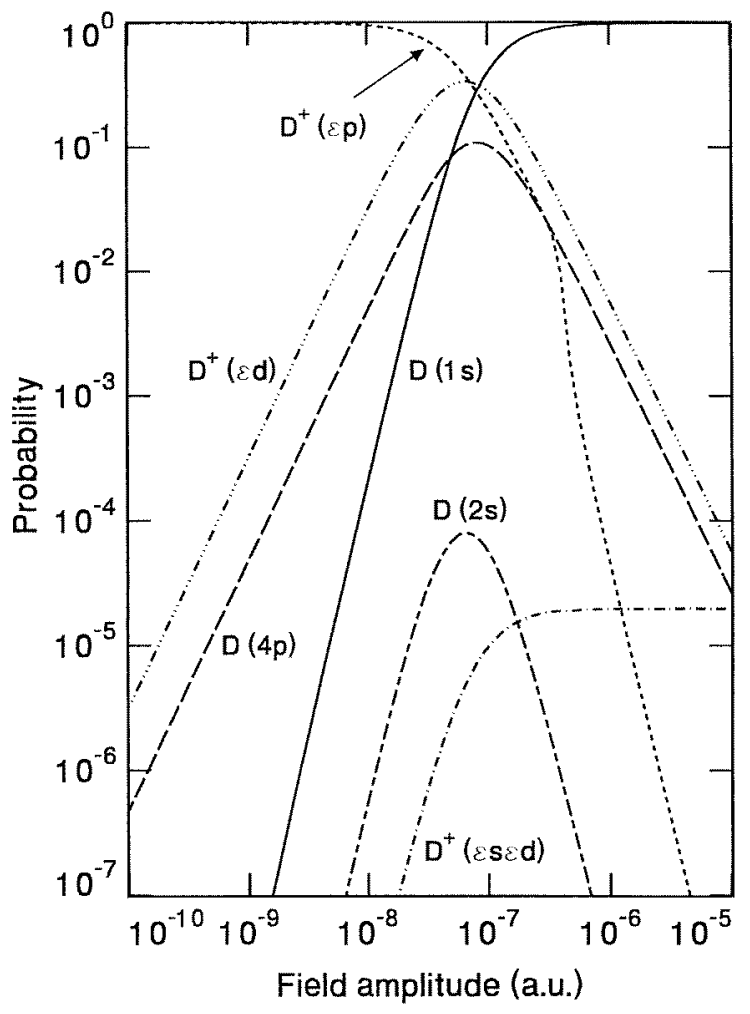

Figure 2. Graph of occupation probabilities as a function of the amplitude of the fundamental field $(486 \mathrm{~nm})$ for monochromatic, rectangular laser pulses of duration $24.2 \mathrm{~ns}\left(10^{9} \mathrm{au}\right)$. The amplitude of the second harmonic field $(243 \mathrm{~nm})$ is held constant at $8 \times 10^{-5}$ au $\left(2.25 \times 10^{8} \mathrm{~W} \mathrm{~cm}^{-2}\right)$ throughout.

least for some values of the fundamental field. Note, however, that averaging over the laser bandwidth smooths the effects of the oscillations.

Any experimentally introduced broadening mechanism (laser bandwidth, Doppler broadening) will smear out the above effects, as will the spatial and temporal variation in intensity experienced by the atoms. In order to compare these predictions with experiment, we have folded the frequency characteristics of our laser system into our theoretical model and the results are discussed in section 4. Other line broadening effects are negligible in our experiment.

\section{Experiment}

The fundamental and second harmonic outputs of an excimer-pumped dye laser (Lambda Physik LEXTRA 50 + LPD 3002) are used in the experiment. The fundamental beam is linearly polarized (10:1) and mutually perpendicular to the linearly polarized second harmonic beam. The maximum pulse energies are 6 and $1.4 \mathrm{~mJ}$, respectively. The temporal shape of the beams is approximately Gaussian with pulse widths of 13 ns FWHM. The bandwidths of the fundamental and the second harmonic beams are $0.18 \mathrm{~cm}^{-1}(5 \mathrm{GHz})$ and $0.36 \mathrm{~cm}^{-1}(10 \mathrm{GHz})$, respectively. The beams are separated by a dichroic mirror $(243 \mathrm{~nm}$ reflected, $486 \mathrm{~nm}$ transmitted) which has an extinction ratio of 100:1. A Pellin-Broca prism 
removes any residual fundamental from the second harmonic beam. The intensity of the fundamental beam is varied using a frustrated internal reflection attenuator. The beams are then recombined using a second dichroic mirror and focused into the centre of the atomic deuterium beam. Molecular deuterium is dissociated in a radio-frequency discharge and effuses through a $1 \mathrm{~mm}$ capillary into the interaction region, realizing an atomic deuterium density of the order of $5 \times 10^{11}$ atoms $/ \mathrm{cm}^{3}$. (Deuterium is used rather than hydrogen because of the somewhat higher dissociation fraction obtained.) The atom and laser beams are perpendicular so as to minimize Doppler broadening. Complete spatial overlap of the laser beams in the interaction region is achieved by using a chromatic lens. The lens has a focal length of $440 \mathrm{~mm}$ at $486 \mathrm{~nm}$ and $400 \mathrm{~mm}$ at $243 \mathrm{~nm}$, giving the fundamental and second harmonic beam diameters of 1 and $0.4 \mathrm{~mm}$, respectively, in the interaction region. The long focal lengths provide a large depth of focus so that the beams are approximately cylindrical in the interaction region.

The $\mathrm{D}^{+}$ions produced in the interaction region are extracted by an electric field of $480 \mathrm{~V} \mathrm{~cm}^{-1}$ applied in the interaction region about $10 \mathrm{~ns}$ after the laser pulse ends. The extraction field has a rise time of $15 \mathrm{~ns}$. The ions are then focused onto a micro-channel plate (MCP) detector after passing through a short drift region for mass discrimination.

The yield of metastable $\mathrm{D}(2 \mathrm{~s})$ atoms is measured by observing the Lyman- $\alpha$ radiation resulting from the quenching of the excited atoms in the extraction field. The $2 s$ and $2 p$ states are fully mixed in the field of $480 \mathrm{~V} \mathrm{~cm}^{-1}$. The Lyman- $\alpha$ radiation is collimated by a magnesium fluoride lens, passes through a Lyman- $\alpha$ filter (transmission $22 \%$ at $121 \mathrm{~nm}$ ) with a $10^{4}: 1$ extinction ratio at $243 \mathrm{~nm}$ and is detected in a CsI-coated MCP located directly above the interaction region. Considerable care has been taken to ensure that the interaction region is well shielded and is free of stray fields during the laser pulse. Both the ion detector and the Lyman- $\alpha$ detector operate in analogue mode and their impedance matched output is recorded by a digital storage oscilloscope which has a bandwidth of $250 \mathrm{MHz}$. We emphasize that the extraction field used to quench the metastable atoms is applied after the laser pulse. In this respect the present experiment differs from the experiments of fieldinduced second harmonic generation of Lyman- $\alpha$ in atomic hydrogen by Hakuta et al (1991, 1992) and Marmet et al (1992) where an electric field was present during the laser pulse.

We have measured the ion and metastable yields as a function of the fundamental field intensity from $350 \mathrm{~W} \mathrm{~cm}{ }^{-2}$ to $88 \mathrm{MW} \mathrm{cm}^{-2}$ (amplitude $\varepsilon_{1}=1 \times 10^{-7}-5 \times 10^{-5} \mathrm{au}$ ), while keeping the second harmonic intensity constant at $65 \mathrm{MW} \mathrm{cm}^{-2}$ (amplitude $\varepsilon_{0}=4.3 \times 10^{-5} \mathrm{au}$ ). Each datum point is the average of 70 laser shots leaving a maximum statistical uncertainty of $<2 \%$ for the laser intensity and $<3 \%$ and $<5 \%$ for the integrated ion and Lyman- $\alpha$ signals, respectively. The intensity of the fundamental has been measured on a shot-to-shot basis with a photodiode (Hamamatsu S1337-66BQ) coupled to an integrating amplifier. These measurements have been calibrated with measurements of the energy (Molectron J9-LP pyroelectric energy meter), the pulse length (New Focus $125 \mathrm{MHz}$ fast photodiode) and focal spot size (lumogen-coated UV-sensitive CCD) at the beginning and end of each experimental run. The total systematic uncertainty in our laser intensity data is $\pm 20 \%$ and is obtained by quadrature addition of the uncertainties in the above measurements.

\section{Results and discussion}

A comparison between the experimental and theoretical results is shown in figure 3 , where the theoretical results data have been averaged over the laser bandwidths. Because the conversion of our integrated experimental signals to occupation probabilities requires an 


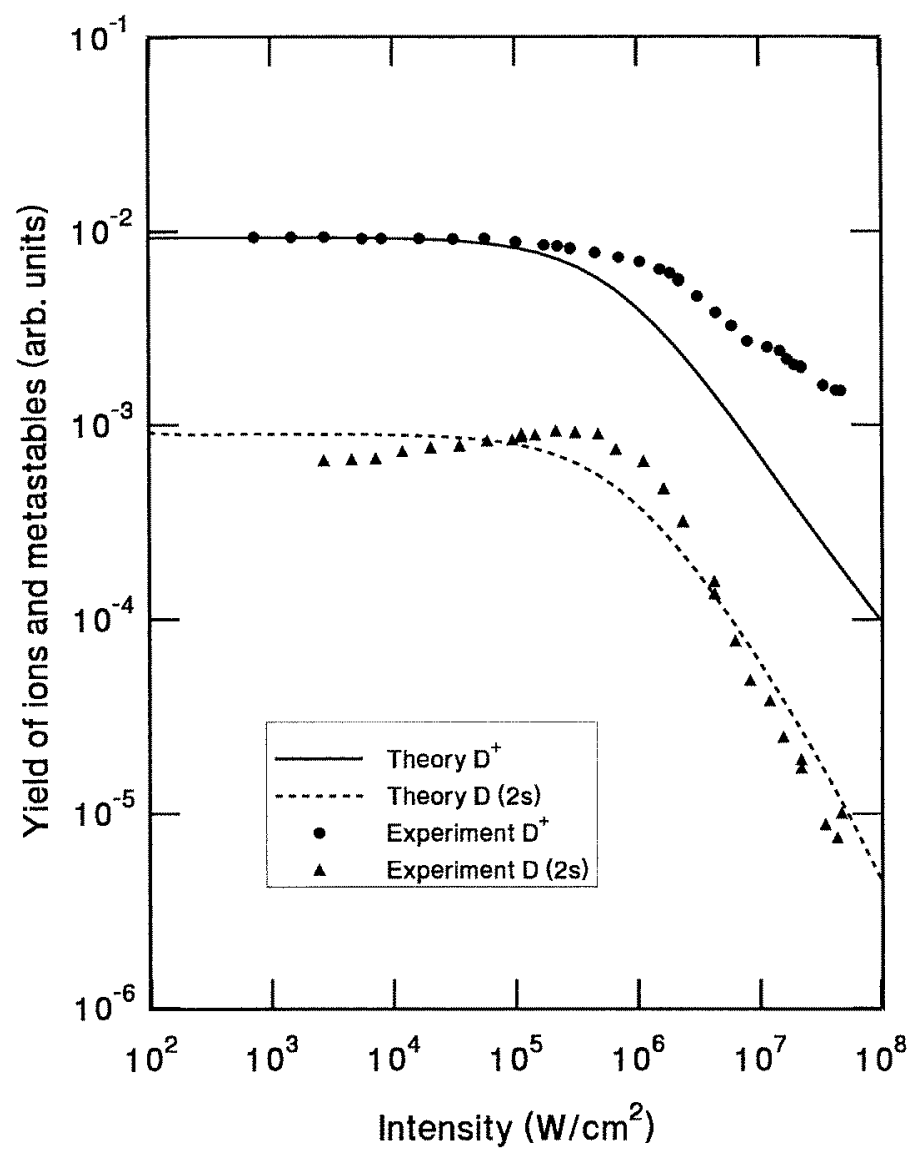

Figure 3. The full and broken curves represent the theoretical total ionization and $2 \mathrm{~s}$ excitation probabilities, respectively. The theory takes into account light fields with bandwidths comparable with the experiment $(5 \mathrm{GHz}$ for the fundamental beam and $10 \mathrm{GHz}$ for the second harmonic beam), and a second harmonic field amplitude of $4.3 \times 10^{-5}$ au (intensity $65 \mathrm{MW} \mathrm{cm}^{-2}$ ). The experimental data, yields of $\mathrm{D}^{+}$ions $(\bullet)$ and quenched $\mathrm{D}(2 \mathrm{~s})$ atoms $(\boldsymbol{\Lambda})$, have been obtained using a second harmonic laser intensity of $65 \mathrm{MW} \mathrm{cm}^{-2}$. The experimental data have been normalized to the theory as discussed in the text.

accurate knowledge of atom densities as well as detector efficiencies, it is not possible to place our data on an absolute scale of occupation probabilities to an accuracy of better than $\pm 80 \%$. In order to compare with theory, therefore, we normalize our ion data to theory in the intensity region below $2 \times 10^{4} \mathrm{~W} \mathrm{~cm}^{-2}$. In the case of the $\mathrm{D}(2 \mathrm{~s})$ data we normalize our data to theory at an intensity of $1.1 \times 10^{5} \mathrm{~W} \mathrm{~cm}^{-2}$.

Averaging over the second harmonic bandwidth results in a lowering of the ionization probability by a factor of about 100 , while averaging over the fundamental bandwidth increases the laser intensity at which ionization suppression occurs by about 100 in comparison with the monochromatic case (figure 2). The theoretical results for $\mathrm{D}(2 \mathrm{~s})$ now show a broad region of intensity of the fundamental laser where the yield of $\mathrm{D}(2 \mathrm{~s})$ atoms is constant before falling off. The decrease in the production of both $\mathrm{D}(2 \mathrm{~s})$ and $\mathrm{D}^{+}$at high fundamental intensities is now very similar. 
There is good qualitative agreement between theory and experiment over most of the range of experimental intensities. The suppression of the ionization and $2 \mathrm{~s}$ excitation rates, by factors of 9 and 100, respectively, as a function of fundamental intensity is clearly visible. The reduction of ionization and $2 \mathrm{~s}$ excitation continues up to the maximum intensity of our laser. The lower rate of suppression of the ion yield in the experiment as compared with theory is probably due to a combination of factors, including the non-uniform spatial and temporal profiles of the laser beams, and a reduction of the collection efficiency due to space-charge effects in the interaction region.

\section{Conclusion}

In summary we have shown theoretically that the multiphoton ionization rate of atomic deuterium via the intermediate $2 \mathrm{~s}$ state is suppressed by seven orders of magnitude by introducing a second laser frequency resonant with the $2 s-4 p$ transition. Experimental suppression has been demonstrated within the limitations of a laser system of moderate bandwidth. By using a laser of narrow bandwidth this system could act as a very effective photoionization switch.

\section{Acknowledgments}

This work has been supported under the Scientific Research Programme of the Irish Research Agency Forbairt and under the TMR programme of the European Union. One of the authors (RWON) gratefully acknowledges receipt of a European Union Human Capital and Mobility Fellowship.

\section{References}

Autler S H and Townes C H 1955 Phys. Rev. 100703

Chen C, Yin Y Y and Elliot D S 1990 Phys. Rev. Lett. 64507

De Boer M P and Muller H G 1992 Phys. Rev. Lett. 682747

Hakuta K, Marmet L and Stoicheff B P 1991 Phys. Rev. Lett. 66596

-1992 Phys. Rev. A 455152

Harris S E, Field J E and Imamoglu A 1990 Phys. Rev. Lett. 641107

Jones R R 1995 Phys. Rev. Lett. 751491

Marmet L, Hakuta K and Stoicheff B P 1992 J. Opt. Soc. Am. B 91038

Protopapas M and Knight P L 1995 J. Phys. B: At. Mol. Opt. Phys. 284459

Shao Y L, Charalambidis D and Fotakis C 1996 J. Phys. B: At. Mol. Opt. Phys. 295377

Sihombing R S D, Katsuragawa M, Zhang G Z and Hakuta K 1996 Phys. Rev. A 541551

Story J G, Duncan D I and Gallagher T F 1993 Phys. Rev. Lett. 703012

Thompson R I, Stoicheff B P, Zhang G Z and Hakuta K 1995 Appl. Phys. B 60 S129

Vrijen R B, Hoogenraad J H, Muller H G and Noordam L D 1993 Phys. Rev. Lett. 703016

Zhang G Z, Hakuta K and Stoicheff B P 1993 Phys. Rev. Lett. 713099

Zhang G Z, Katsuragawa, Hakuta K, Thompson R I and Stiocheff B P 1995 Phys. Rev. A 521584 